\title{
Farnesol induces apoptosis of DU145 prostate cancer cells through the PI3K/Akt and MAPK pathways
}

\author{
JIN SOO PARK, JUNG KI KWON, HYE RI KIM, HYEONG JIN KIM, BYEONG SOO KIM and JI YOUN JUNG \\ Department of Companion and Laboratory Animal Science, Kongju National University, \\ Yesan, Chungnam 340702, Republic of Korea
}

Received October 7, 2013; Accepted February 13, 2014

DOI: $10.3892 / \mathrm{ijmm} .2014 .1679$

\begin{abstract}
The aim of this study was to investigate the effect of farnesol on the induction of apoptosis in DU145 prostate cancer cells. 3-(4,5-Dimethylthiazol-2-yl)-2,5-diphenyl tetrazolium bromide assay showed that cell proliferation decreased significantly in a dose- and time-dependent manner. 4',6-Diamidino-2-phenylindole staining showed that chromatin condensation in cells treated with $60 \mu \mathrm{M}$ of farnesol was markedly higher than in the control groups. Farnesol increased the expression of p53, p-c-Jun N-terminal kinase, cleaved-caspase-3, Bax, and cleaved-caspase-9, but decreased the expression of p-phosphatidylinositol-3-kinase (PI3K), p-Akt, p-p38, Bcl-2, and p-extracellular signal-regulated protein kinase, in a dose-dependent manner. The apoptotic cell ratio increased in a dose-dependent manner. The tumor growth inhibitory effect of farnesol was investigated in a mouse model. Compared to the control group, tumor volume decreased significantly in the group administered $50 \mathrm{mg} / \mathrm{kg}$ farnesol. Apoptosis was frequently detected in this same group by terminal deoxynucleotidyl transferase dUTP nick-end labeling assay. The results indicated that farnesol induced apoptosis of DU145 prostate cancer cells through the PI3K/ Akt and mitogen-activated protein kinase signaling pathways.
\end{abstract}

\section{Introduction}

A high incidence of lung, breast, prostate, and colorectal cancers has been previously reported (1). Prostate cancer continues to be a major problem in developing countries, the United States and Europe $(2,3)$. In western countries it is a common malignancy and the third most frequently diagnosed form of cancer in males $(1,3)$. In the United States, prostate

Correspondence to: Professor Ji-Youn Jung or Professor ByeongSoo Kim, Department of Companion and Laboratory Animal Science, Kongju National University, Yesan, Chungnam 340-702, Republic of Korea

E-mail: wangza@kongju.ac.kr

E-mail: bskim@kongju.ac.kr

Key words: apoptosis, farnesol, mitogen-activated protein kinase, phosphatidylinositol-3-kinase/Akt, prostate cancer cell cancer is the second leading cause of cancer mortality in males (4). In 2012 an estimated 241,740 males in the United States were diagnosed with prostate cancer, and 28,660 of those cases resulted in death (5). Prostate cancer also accounts for $24 \%$ of new case diagnoses and $13 \%$ of cancer deaths in the United Kingdom (6). Although the pathogenesis of prostate cancer has not yet been determined, some known contributing risk factors include age, ethnicity and diet (7).

Developing prostate cancers require immediate therapies due to their androgen dependency $(8,9)$. However, 18-36 months after initial treatments most patients with prostate cancer become castration-resistant to androgen deprivation therapy, leading to metastasis and the development of hormone-refractory prostate cancer $(2,10)$. When prostatic cancer progresses from being androgen-dependent to -independent, the patient usually succumbs to the disease (11). Androgen-refractory prostate cancer is chemoresistant and grows prior to androgen-dependent cancer $(12,13)$. For this reason, the development of innovative and effective therapeutic options that inhibit androgen-dependent and -independent prostate cancers is needed $(3,14)$.

Isoprenoids are important for cell proliferation, apoptosis, cell differentiation, lipid biosynthesis, and cell cycle arrest (15-24). Sterol and non-sterol isoprenoids are essential for a number of cell functions, including cell signaling, protein synthesis, maintaining membrane integrity, cell proliferation and apoptosis (22). Both types of isoprenoid are natural products produced from a commonplace precursor, mevalonate. Farnesol is a non-sterol isoprenoid produced by the dephosphorylation of farnesol pyrophosphate, a catabolite of the cholesterol biosynthetic pathway $(22,23)$.

Farnesol is a 15-carbon isoprenoid found in a variety of plant products, such as orange peel, lemon-grass oil, strawberries and chamomile (25-27). Farnesol has been shown to have anti-bacterial $(28,29)$, anticancer $(16,21,30,31)$ and olfactory nerve effects (32). The anticancer action of farnesol has been reported to be mediated by farnesoid X receptor activity (33), wherein it induces the expression of thyroid hormone receptor- $\beta 1$ mRNA, regulates gene transcription (34), and facilitates the activities of peroxisome proliferator-activated receptor- $\alpha$ and $-\gamma(35)$.

The phosphatidylinositol-3-kinase (PI3K)/serine/threonine kinase (Akt) signaling pathway is essential for the mediation of several cell activities, including cell proliferation, growth, the inflammatory response, chemotaxis, survival and apop- 
tosis $(36,37)$. Increased activation of the PI3K/Akt signaling pathway is involved in the development of malignant tumors and resistance to chemotherapy (38). In addition, activation of Akt frequently occurs in endometrial and pancreatic cancers and has been linked to a poor prognosis (39-42). In vitro and in vivo studies have reported that inhibition of the PI3K/Akt signaling pathway is important for the success of chemotherapy-induced apoptosis of pancreatic cancer cells (43-45).

Mitogen-activated protein kinases (MAPKs) are serine/ threonine-specific. They interrupt intracellular signaling related to a variety of activities, including cell proliferation, cell survival, cell death and transformation $(46,47)$. MAPKs respond to extracellular stimuli such as growth factors, cytokines, and stress (48). The activities of the major members of the MAPK family, i.e., mitogen-activated protein kinase (p38), extracellular signal-regulated protein kinase 1/2 (ERK1/2), and c-Jun $\mathrm{N}$-terminal kinase (JNK), are regulated by different MAPK cascades (49).

The primary aim of this study was to identify the mechanism of farnesol-induced apoptosis in DU145 prostate cancer cells. Analysis of cell viability by 4',6-diamidino-2-phenylindole (DAPI) staining and Annexin V/propidium iodide (PI) staining determined that farnesol-induced death of DU145 prostate cancer cells was due to apoptosis. The protein levels of phosphorylated JNK, phosphorylated ERK, phosphorylated p38, p53, Bcl-2, Bax, cleaved caspase-3, and phosphorylated Akt were also determined. In vivo, tumor size was measured twice per week for up to 5 weeks. Farnesol-induced cell death was also examined by the terminal deoxynucleotidyltransferase-mediated dUTP nick-end labeling (TUNEL) assay.

\section{Materials and methods}

Cells, materials, antibodies and reagents. Human prostate cancer DU145 cells were obtained from the Korean Cell Line Bank. Farnesol (Fig. 1) and 3-(4,5-dimethyl-2-yl)-2,5diphenyl tetrazolium bromide (MTT) were purchased from Sigma-Aldrich (St. Louis, MO, USA). RPMI-1640 medium, trypsin-EDTA, penicillin-streptomycin, and fetal bovine serum (FBS) were obtained from HyClone Laboratories (Logan, UT, USA). Primary antibodies were purchased from Cell Signaling Technology (Danvers, MA, USA). Dimethyl sulfoxide (DMSO) was purchased from Merck (Darmstadt, Germany). Tween-20 was purchased from Sigma-Aldrich. Cell lysis buffer and DAPI were obtained from Invitrogen (Carlsbad, CA, USA). A fluorescein isothiocyanate (FITC)conjugated Annexin $\mathrm{V}$ apoptosis detection kit was purchased from BD Biosciences (San Diego, CA, USA).

Preparation of farnesol and cell culture. Farnesol was dissolved in a 1:14.6 mixture of Tween-20 and DMSO prior to administration. Final concentrations ranged from 0 to $100 \mu \mathrm{M}$. Test agents were added to the culture medium at concentrations of $0,10,20,30,40,50,60,70,80,90$ and $100 \mu \mathrm{M}$ with a final DMSO concentration of $<0.1 \%$.

DU145 cells were cultured in RPMI-1640 supplemented with $10 \%$ FBS, $100 \mu \mathrm{g} / \mathrm{ml}$ penicillin and $100 \mu \mathrm{g} / \mathrm{ml}$ streptomycin in $5 \% \mathrm{CO}_{2}$ at $37^{\circ} \mathrm{C}$. Cells were then subcultured in a $175-\mathrm{cm}^{2}$ flask (Nunc, Fisher Scientific, Loughborough, UK) at a density of $80-90 \%$.
Cell viability assay. The effect of farnesol on DU145 cell survival was determined by MTT assay. Cells were seeded in 96-well microplates at two densities: wells containing $2 \times 10^{4}$ cells $/ \mathrm{ml}$ were incubated for $24 \mathrm{~h}$ and those containing $1 \times 10^{4}$ cells $/ \mathrm{ml}$ were incubated for $48 \mathrm{~h}$. The two groups underwent treatment with $0-100 \mu \mathrm{M}$ farnesol. Following incubation, the medium was removed and the cells were incubated for $2 \mathrm{~h}$ with $40 \mu \mathrm{l}$ of a solution containing $5 \mathrm{mg} / \mathrm{ml}$ MTT in phosphatebuffered saline (PBS). Then, $100 \mu 1$ of DMSO was added to each well to dissolve the formazan crystal. Absorbance at $570 \mathrm{~nm}$ was measured using an ELISA reader (Bio-Rad, Hercules, CA, USA). Cell viability was expressed as the ratio of optical density values of the treatment and control groups.

Nuclear morphology. To assess the extent of apoptosis, DU145 cell nuclei were stained with DAPI and observed using fluorescence microscopy. DU145 cells were seeded in 12-well microplates at a density of $4 \times 10^{4}$ cells/well and incubated for $24 \mathrm{~h}$ with 0,30 or $60 \mu \mathrm{M}$ farnesol. Following treatment the cells were fixed and incubated in PBS containing 4\% paraformaldehyde for $30 \mathrm{~min}$. After fixation, the cells were washed twice with PBS and nuclei were stained with DAPI. Fluorescence signals were visualized using a fluorescence microscope at a x200 magnification.

Western blotting. DU145 cells were incubated with 0,30 or $60 \mu \mathrm{M}$ farnesol for $24 \mathrm{~h}$. Cells were harvested by washing the flasks once with ice-cold PBS and then treating cells for $3 \mathrm{~min}$ with trypsin-EDTA. Floating cells were collected, transferred to $50-\mathrm{ml}$ conical centrifuge tubes, and centrifuged for $7 \mathrm{~min}$ at $260 \mathrm{x}$ g. The resulting cell pellets were washed twice with PBS and resuspended in $200 \mu \mathrm{l}$ of lysis buffer. Lysates were prepared and centrifuged at $15,920 \mathrm{x} \mathrm{g}$ for $5 \mathrm{~min}$ at $4^{\circ} \mathrm{C}$.

Protein concentrations were determined by a Bradford protein assay (Bio-Rad). Protein $(30 \mu \mathrm{g})$ was separated by $12 \%$ SDS-PAGE and transferred electrophoretically to polyvinylidene difluoride membranes (Amersham Biosciences). The transferred membranes were blocked with Tris-buffered saline containing $5 \%$ non-fat dry milk and $0.1 \%$ Tween- 20 for $2 \mathrm{~h}$ at $4^{\circ} \mathrm{C}$. After blocking, but prior to incubation, the blocked membranes were treated individually with the following antibodies: anti- $\beta$-actin, anti-cleaved caspase- 3 , anti-cleaved caspase-9, anti-Bcl-2, anti-Akt, anti-phospho-Akt, anti-Bax, anti-phospho-PI3K, anti-phospho-JNK, anti-phospho-ERK, anti-phospho-p38 and anti-p53. Membranes were then incubated overnight with gentle agitation at $4^{\circ} \mathrm{C}$.

Following incubation the membranes were washed with Tris-buffered saline containing $0.1 \%$ Tween-20, treated with secondary antibodies (horseradish peroxidase-conjugated goat anti-mouse or -rabbit IgG; Cell Signaling Technology), and incubated with gentle agitation at room temperature for $2 \mathrm{~h}$. The membranes were washed once more with Tris-buffered saline containing $0.1 \%$ Tween-20, and bands were subsequently visualized using the ECL detection reagents (Pierce, Rockford, IL, USA).

Apoptosis analysis. Following treatment with farnesol, DU145 cell apoptosis was measured using an Annexin V staining kit (Becton-Dickinson, Franklin Lakes, NJ, USA). Cells were incubated with 0,30 or $60 \mu \mathrm{M}$ farnesol for $24 \mathrm{~h}$ and harvested 


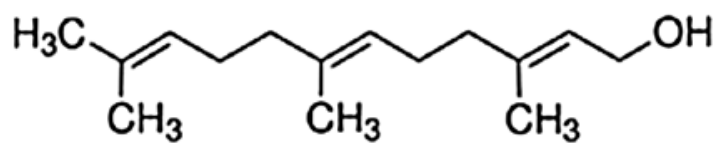

Figure 1. The structure of farnesol $\left(\mathrm{C}_{14} \mathrm{H}_{12} \mathrm{O}_{3}\right.$, molecular weight: 222.37).

by exposure to trypsin-EDTA for $3 \mathrm{~min}$. The harvested cells were washed once with PBS, resuspended in $100 \mu \mathrm{l}$ of Annexin $\mathrm{V}$ binding buffer, and mixed with $5 \mu \mathrm{l}$ of fluorescein isothiocyanate-conjugated Annexin V and phycoerythrinconjugated PI. The resuspended cells were then incubated at room temperature in the dark for $15 \mathrm{~min}$. The frequency of apoptotic cells was analyzed using a FACSCalibur ${ }^{\mathrm{TM}}$ flow cytometer (Becton-Dickinson).

Animals. Five-week-old male nude mice weighing 20-30 g were purchased from Orient Bio (Gyeonggi-do, Korea). They were maintained at $23 \pm 5^{\circ} \mathrm{C}$ and a relative humidity of $40 \pm 10 \%$ with artificial lighting from from 800 to 2,000 lx in an animal laboratory approved by the Companion and Laboratory Animal Science Department of Kong-Ju National University. The mice were housed in cages and allowed free access to autoclaved water, food and commercial rodent chow (Biopia, Seoul, Korea). Animal experiments were performed with the approval of the Institutional Animal Care and Use Committee and according to the guidelines of Kong-Ju National University.

Xenografts. DU145 cells were grown in media composed of RPMI-1640 supplemented with $10 \%$ FBS, $100 \mu \mathrm{g} / \mathrm{ml}$ penicillin and $100 \mu \mathrm{g} / \mathrm{ml}$ streptomycin. The cells were then harvested by washing with ice-cold PBS and exposure to trypsin-EDTA. Collected cells were washed twice with PBS and resuspended in RPMI-1640 medium.

DU145 prostate cancer cells at a density of $5 \times 10^{6}$ cells/0.2-ml medium were injected subcutaneously into the flanks of donor nude mice. When the resulting tumors reached $2,000 \mathrm{~mm}^{3}$ in size, the mice were anesthetized with diethyl ether and the tumor masses were removed surgically. After slicing the mass into $2 \times 2-m m$ sections, tumor fragments were implanted subcutaneously into the right flank of each mouse. After tumor implantation, mice were randomly divided into two groups ( $n=3$ each).

Preparation and administration of farnesol. Three mice were divided randomly into different treatment groups. Oral treatment of each group began on the day of tumor fragment implantation and continued for 5 weeks. Control mice received $0.2-\mathrm{ml}$ corn oil daily for 5 weeks. Mice in the treatment group received $50 \mathrm{mg} / \mathrm{kg}$ farnesol daily for 5 weeks.

Measurement of tumor size. Mice were observed for 5 weeks following tumor implantation. Tumor sizes were measured twice per week using vernier calipers (Mitutoyo, Kawasaki, Japan). Tumor sizes were calculated as: width ${ }^{2} \mathrm{x}$ length/2.

TUNEL assay. Apoptotic cells were detected using the Dead End $^{\mathrm{TM}}$ Colorimetric TUNEL System (Promega, Madison, WI, USA). Tumor paraffin blocks were separated into $5-\mu \mathrm{m}$

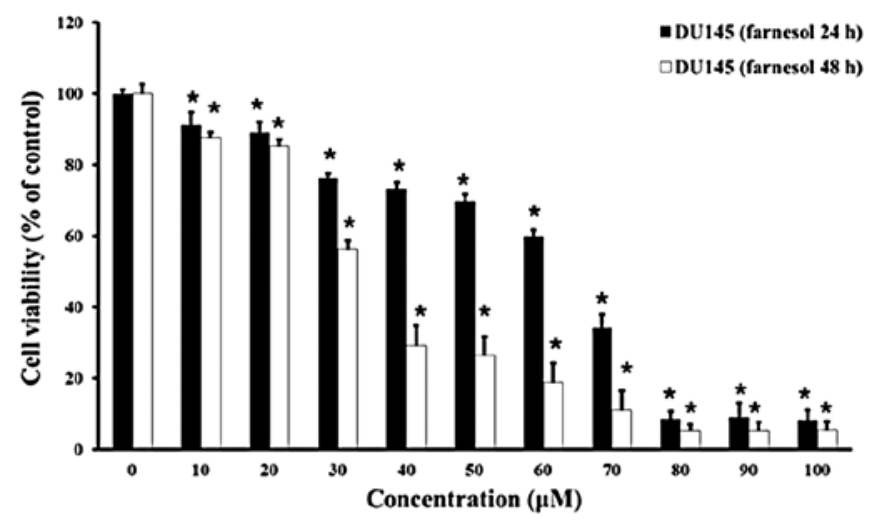

Figure 2. The effect of farnesol on the proliferation of DU145 prostate cancer cells. Cell suspensions of $2 \times 10^{4}$ and $1 \times 10^{4}$ cells $/ \mathrm{ml}$ were treated with $0-100 \mu \mathrm{M}$ farnesol for 24 or $48 \mathrm{~h}$. Cell viability was measured by MTT assay. Values are means \pm standard deviation (SD). Significance was determined by Dunnett's t-test $(\mathrm{P}<0.05)$.

sections using a microtome (Shandon Inc., Pittsburgh, PA, USA) and attached to microscope slides.

After washing in running water overnight, slides were deparaffinized by immersion in fresh xylene. The slides were then washed with $100 \%$ ethanol and the tumor tissues were rehydrated by soaking in a graded ethanol series. The samples were washed with PBS for $5 \mathrm{~min}$ and treated with $20 \mu \mathrm{g} / \mathrm{ml}$ proteinase for $20 \mathrm{~min}$ at room temperature. Endogenous peroxidase was inactivated by $0.3 \% \mathrm{H}_{2} \mathrm{O}_{2}$ in PBS for $5 \mathrm{~min}$. The samples were then washed with PBS for $5 \mathrm{~min}$ and the slides were treated with streptavidin HRP in PBS for $30 \mathrm{~min}$.

The TUNEL sections were visualized using 3,3'-diaminobenzidine tetrahydrochloride (DAB) solution. A total of $50 \mu \mathrm{l}$ of 20X DAB substrate buffer was added to $950 \mu 1$ of deionized water, $50 \mu \mathrm{l}$ of $20 \mathrm{X}$ DAB chromogen and $50 \mu \mathrm{l}$ of $20 \mathrm{X}$ hydrogen peroxide. This DAB solution $(100 \mu \mathrm{l})$ was added to each slide and the sections were stained with methyl green. Randomly selected fields were visualized under a light microscope at a x400 magnification. The number of cells positively stained by each antibody was counted.

Statistical analysis. Data were expressed as the means \pm standard deviation (SD). One-way analysis of variance was used to analyze differences in multiple comparisons. $\mathrm{P}<0.05$ was considered to indicate statistical significance.

\section{Results}

Farnesol-induced inhibition of cell proliferation. Treatment with farnesol results in inhibition of the proliferation of DU145 cells. The chemical structure of farnesol is shown in Fig. 1.

To determine the effect of farnesol on their growth, DU145 cells were treated with $0-100 \mu \mathrm{M}$ farnesol for 24 or $48 \mathrm{~h}$, and viability was determined by MTT assay (Fig. 2).

Farnesol decreased the viability of DU145 cells in a doseand time-dependent manner. In comparison to the control group, cell viability began to decrease significantly at $10 \mu \mathrm{M}$ in the 24- and 48-h groups $(\mathrm{P}<0.05)$. The resulting cell viabilities after treatment with 30,60 or $100 \mu \mathrm{M}$ farnesol were $76.3 \pm 1.1$, $60.0 \pm 1.7$ and $8.1 \pm 2.8 \%$, respectively , at $24 \mathrm{~h}$; and $56.3 \pm 2.3$, 
A

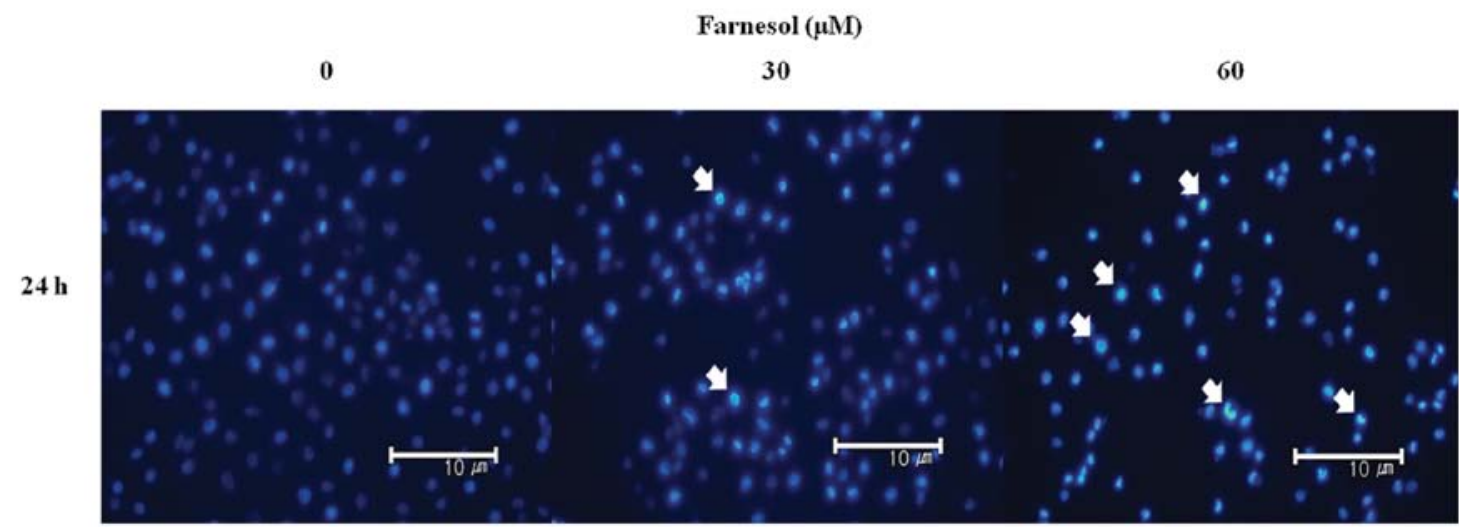

B

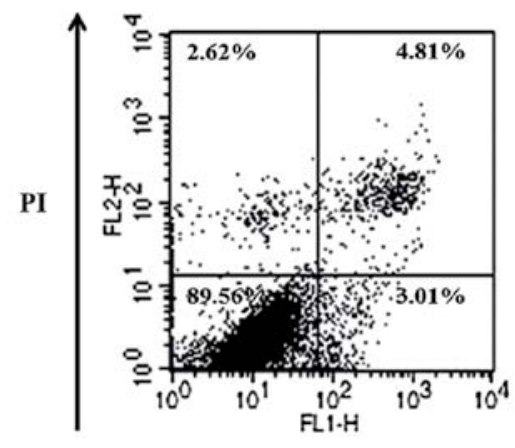

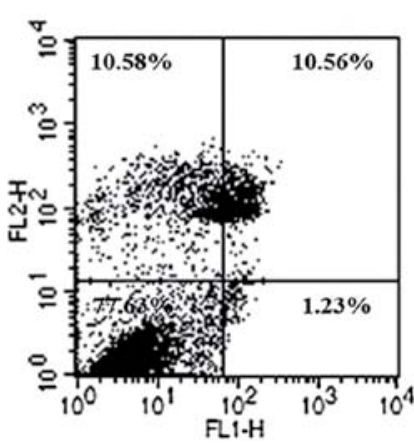

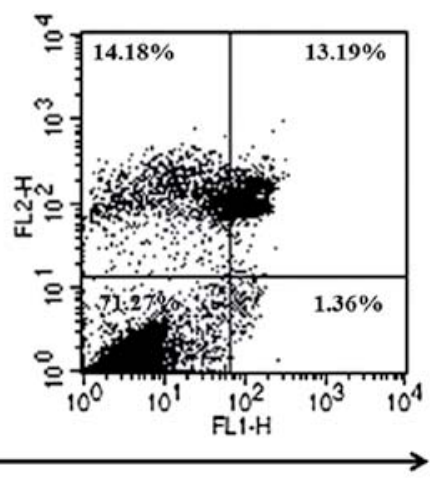

Annexin V-FITC

Figure 3. The effect of farnesol in DU145 cells as assessed by 4',6-diamidino-2-phenylindole (DAPI) staining and Annexin-positive apoptotic cell numbers. (A) DU145 cells were treated with 0,30 or $60 \mu \mathrm{M}$ farnesol for $24 \mathrm{~h}$ and apoptotic bodies were identified by DAPI staining. The mean \pm standard deviation (SD) of triplicate separated experiments is shown. Arrows indicate chromatin condensation in DU145 cells. Cleaved nuclei were visualized using a fluorescence microscope at a x200 magnification. (B) DU145 cells were treated with 0, 30 or $60 \mu \mathrm{M}$ farnesol for $24 \mathrm{~h}$. Apoptosis was assayed by Annexin V/PI double staining and FACS. The mean \pm standard deviation (SD) of triplicate independent experiments is shown.

$18.8 \pm 5.4$ and $5.4 \pm 2.3 \%$, respectively, at $48 \mathrm{~h}$. Farnesol concentrations $>80 \mu \mathrm{M}$ for 24 or $48 \mathrm{~h}$ resulted in an $\sim 90 \%$ reduction in cell viability.

These results suggested that farnesol induces cell death in DU145 cells in a dose- and time-dependent manner. According to the MTT assay, 30 and $60 \mu \mathrm{M}$ farnesol were used in subsequent experiments.

Farnesol increases chromatin condensation in DU145 prostate cancer cells. The cell proliferation results suggested that the number of dead cells increased in a dose- and time-dependent manner after farnesol treatment (Fig. 2). Chromatin condensation increased following treatment with 30 or $60 \mu \mathrm{M}$ farnesol.

DAPI staining distinguishes viable and apoptotic cells based on nuclear morphology. To investigate the chromatin condensation effects, DU145 cells were treated with 30 or $60 \mu \mathrm{M}$ farnesol for $24 \mathrm{~h}$ and chromatin condensation was visualized by DAPI staining. Changes in the morphology of farnesol-treated DU145 cells were detected using a fluorescence microscope at a x200 magnification (Fig. 3A). Chromatin condensation was detected in DU145 cells treated with 30 or $60 \mu \mathrm{M}$ farnesol in a dose-dependent manner. Characteristic apoptotic cell features were confirmed in DU145 cells treated with farnesol, including cytoplasmic condensation, loss of cell volume, nuclear fragmentation, convoluted nuclei, and apoptotic bodies. The untreated group did not exhibit features of apoptosis (Fig. 3A). These
DAPI staining results suggested that farnesol induces apoptosis of DU145 cells in a dose-dependent manner.

Farnesol induces apoptosis in DU145 prostate cancer cells. The cause of farnesol-induced cell death was identified as apoptosis or necrosis. Apoptosis of DU145 cells treated with 30 or $60 \mu \mathrm{M}$ farnesol was confirmed by Annexin V/PI double staining. As shown in Fig. 3B, farnesol induced apoptosis in DU145 cells in a dose-dependent manner. At $24 \mathrm{~h}$ treatment with 30 and $60 \mu \mathrm{M}$ farnesol induced apoptosis in 11.79 and $14.55 \%$ of cells, respectively. Flow cytometry confirmed that farnesol induces apoptosis of DU145 prostate cancer cells.

Farnesol induces apoptosis through the PI3K/Akt pathway. The effect of farnesol on the PI3K/Akt pathway was subsequently examined. DU145 cells were treated with 30 or $60 \mu \mathrm{M}$ farnesol for $24 \mathrm{~h}$ and apoptosis-related proteins assayed by western blotting. As shown in Fig. 4, PI3K phosphorylation was lower in the farnesol-treated group compared to the control group. The farnesol treatment group exhibited decreased Akt and Bcl-2 phosphorylation (Fig. 4).

By contrast, Bax, cleaved caspase- 9 and cleaved caspase- 3 levels increased in the farnesol-treated group (Fig. 4). These results suggested that farnesol induced apoptosis via inhibition of the PI3K/Akt pathway and activation of the caspase chain reaction. 


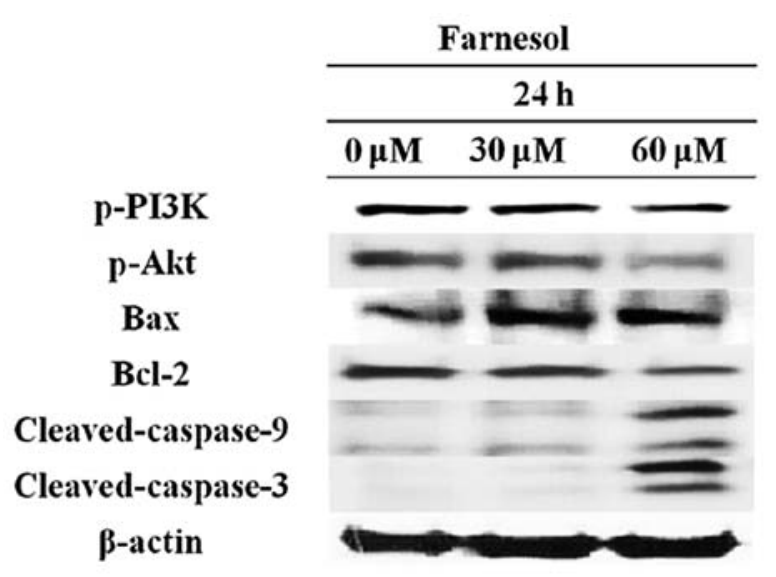

Figure 4 . The effect of farnesol on the apoptotic pathway. Cellis were treated with 0,30 or $60 \mu \mathrm{M}$ farnesol for $24 \mathrm{~h}$. Lysates were then resolved by $12 \%$ SDS-PAGE, followed by western blotting using anti-phospho-phosphatidylinositol-3-kinase (PI3K), phospho-Akt, Bax, Bcl-2, cleaved-caspase-9, and cleaved-caspase- 3 antibodies. The mean \pm standard deviation (SD) of multiple independent experiments is shown.

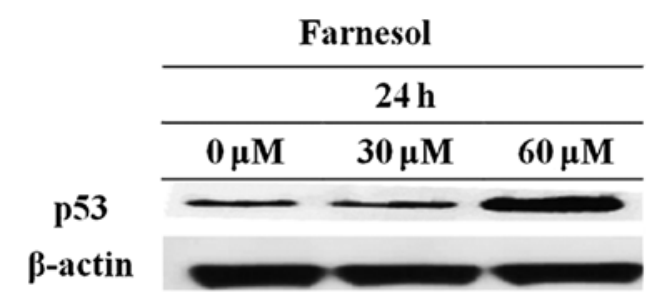

Figure 5. The effect of farnesol on p53. Cells were treated with 0,30 or $60 \mu \mathrm{M}$ farnesol for $24 \mathrm{~h}$. Lysates were then resolved by 12\% SDS-PAGE, followed by western blotting using an anti-p53 antibody. The mean \pm standard deviation (SD) of multiple independent experiments is shown.

Farnesol increases tumor suppressor gene p53 expression. p53 arrests the cell cycle $(50,51)$ and induces apoptosis $(52,53)$. p53 induces apoptosis by increasing the expression of apoptosis-related proteins (54-56). DU145 cells were treated with 30 or $60 \mu \mathrm{M}$ farnesol for $24 \mathrm{~h}$ and the 533 level was assayed by western blotting. p53 expression was increased in the farnesoltreated group compared to the control group (Fig. 5).

Farnesol regulates the expression of MAPK pathway genes in DU145 prostate cancer cells. The three main MAPK members are stress-activated protein kinase JNK, stress-activated protein kinase $2(\mathrm{p} 38)$, and ERK. MAPKs are associated with a variety of cellular activities.

The p38 MAPK signaling pathway is activated in cancer cells. Therefore, carcinostatic substances should inhibit activation of the p38 MAPK pathway. DU145 cells were treated with 30 or $60 \mu \mathrm{M}$ farnesol for $24 \mathrm{~h}$ and p-JNK, p-p38 and p-ERK levels were determined by western blotting. As shown in Fig. 6 , p-JNK expression was increased in the farnesol-treated group compared to the control group. However, the treatment group exhibited decreased levels of $\mathrm{p} 38$ and p-ERK phosphorylation. These results indicated that farnesol induced apoptosis via regulation of the MAPK pathway.

Farnesol inhibits the growth of DU145 prostate tumors. The apoptosis-inducing effects of farnesol in DU145 cells were

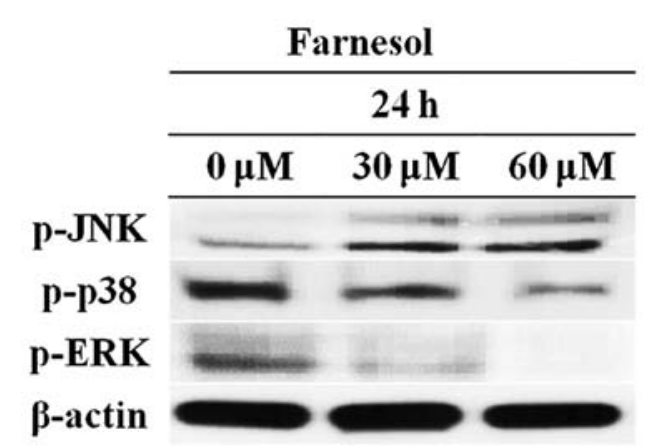

Figure 6. The effect of farnesol on the mitogen-activated protein kinase (MAPK) pathway. Cells were treated with 0,30 or $60 \mu \mathrm{M}$ farnesol for $24 \mathrm{~h}$. Lysates were then resolved by $12 \%$ SDS-PAGE, followed by western blotting using anti-phospho-c-Jun N-terminal kinase (JNK), phospho-p38 (p-p38), and phospho-extracellular signal-regulated protein kinase (p-ERK) antibodies. The mean \pm standard deviation (SD) of multiple independent experiments is shown.

confirmed prior to in vivo investigations using an animal model. To assess the antitumor effect of farnesol, a mouse xenograft model with DU145 prostate cancer cells was used. After subcutaneous inoculation of DU145 cells into athymic nude mice, farnesol was administered for 5 weeks and tumor sizes were measured at 2-day intervals. As shown in Fig. 7A, the tumor volume in animals treated with $50 \mathrm{mg} / \mathrm{kg}$ farnesol was reduced by $50 \%$ compared with the control group $(\mathrm{P}<0.05)$. The average tumor volumes at 5 weeks were $1,000 \mathrm{~mm}^{3}$ in the control group and $800 \mathrm{~mm}^{3}$ in the farnesol group (Fig. 7A). Administration of farnesol reduced tumor weights compared to the control group (Fig. 7B).

Farnesol induces apoptosis in DU145 prostate tumors. The effect of farnesol was studied in two groups of mice. When tumors in control mice reached $2,000 \mathrm{~mm}^{3}$, the animals in the two groups were sacrificed and 5- $\mu \mathrm{M}$ sections were prepared. As shown in Fig. 7C, a significant increase in the number of TUNEL-positive cells was observed in mice treated with $50 \mathrm{mg} / \mathrm{kg}$ farnesol compared to the control group $(\mathrm{P}<0.05)$.

\section{Discussion}

Farnesol has been reported to induce cell death in numerous cancer cell lines $(16,21,30,31)$. Several studies have demonstrated that farnesol also has anti-bacterial effects $(28,29)$. However, the effect of farnesol in DU145 prostate cancer cells is not well understood. This study investigated the anticancer effects of farnesol in DU145 prostate cancer cells mediated by PI3K/Akt and MAPK signaling. Farnesol induced dose- and time-dependent apoptosis in DU145 prostate cancer cells.

To assess the effect of farnesol on the viability of DU145 cells, the MTT assay was performed. Farnesol significantly inhibited the growth of DU145 prostate cancer cells in a doseand time-dependent manner (Fig. 2).

Apoptosis occurs as the result of a highly complex cascade of cellular events characterized by cell shrinkage, chromatin condensation and cell fragmentation $(57,58)$. To investigate the effect of farnesol on chromatin condensation, DAPI staining 
A

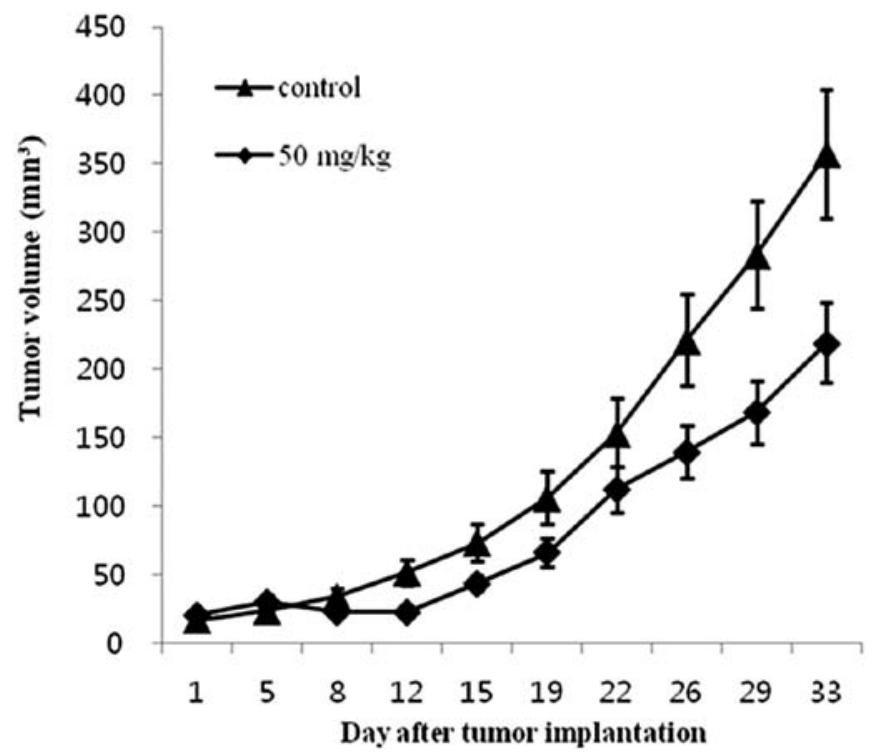

B

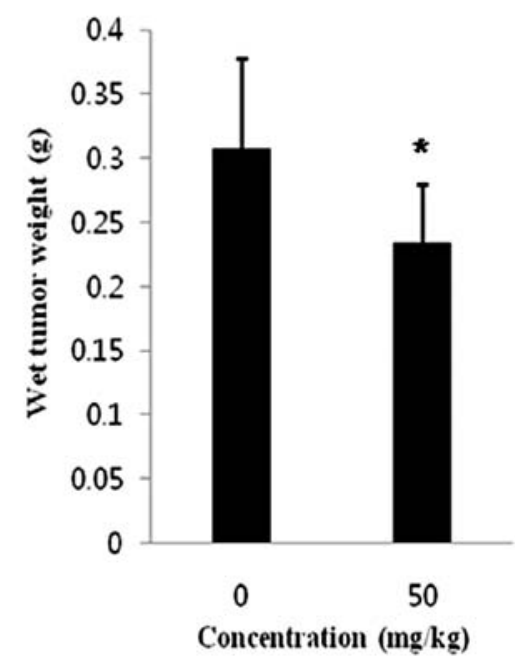

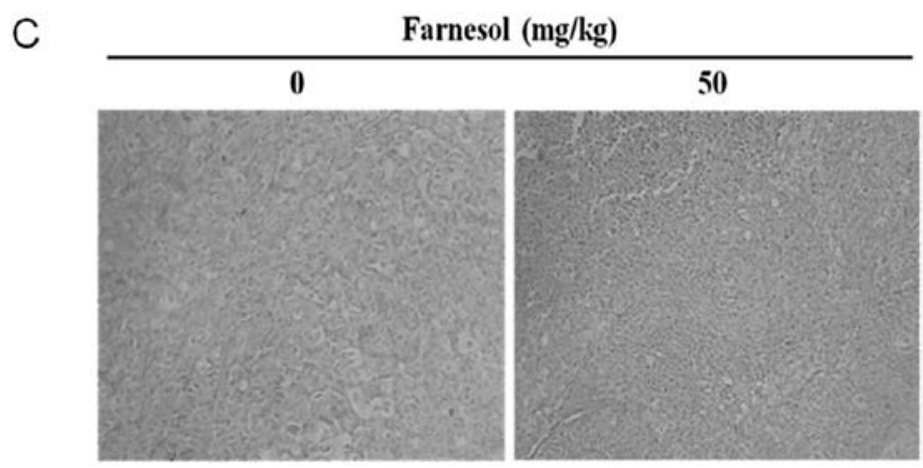

Figure 7. The inhibition of DU145 prostate tumor growth and enhancement of apoptosis in DU145 prostate tumors by farnesol. A xenograft experiment was performed as described. (A) To identify its effect on DU145 prostate tumor growth, nude mice were treated with farnesol for 29 days ( $\mathrm{n}=5$, $\mathrm{P}<0.05$ ). (B) Final tumor weights are shown. Data are presented as means \pm standard (SD) deviation of 5 mice, $\mathrm{P}<0.05$. (C) Nude mice were treated with farnesol for 29 days and apoptosis was assessed by terminal deoxynucleotidyltransferase-mediated dUTP nick-end labeling (TUNEL) assay. Tumor tissues were observed under a microscope and photographed at a x200 magnification. Paraffin-embedded tumors were cut into 5- $\mu \mathrm{m}$ sections. Scale bar, $10 \mu \mathrm{m}$.

was performed. As shown in Fig. 3A, the frequency of apoptotic bodies increased in the farnesol treatment group compared to the untreated group. Cells treated with $60 \mu \mathrm{M}$ farnesol fluoresced more intensely, indicating chromatin condensation. Thus, farnesol the potentially able to induce apoptosis.

The results of Annexin V/PI staining were similar. As shown in Fig. 3B, 24-h treatment with 30 and $60 \mu \mathrm{M}$ farnesol induced apoptosis in 11.79 and $14.55 \%$ of the cells, respectively. These results indicate that farnesol increased apoptosis in a dose-dependent manner.

Western blot assays revealed that treatment of DU145 cells with farnesol altered the expression of PI3K/Akt pathway proteins. As shown in Fig. 4, farnesol treatment inhibited Akt and PI3K phosphorylation and decreased the level of Bcl-2. By contrast, Bax, cleaved caspase- 3 and cleaved caspase- 9 expression increased compared to the control group (Fig. 4), suggesting that farnesol induces apoptosis by altering the expression of PI3K/Akt pathway proteins.

p53 is a well-known tumor suppressor gene. Upregulation of p53 is associated with significant inhibition of Akt phosphorylation (59). As shown in Fig. 5, p53 activity increased following farnesol treatment. Therefore, farnesol induced apoptosis by the inhibition of p-Akt.

MAPKs are associated with a variety of cell activities, including cell proliferation, survival, death, and transformation $(46,47)$. Western blotting was performed to determine the involvement of the MAPK pathway in farnesol-induced apoptosis. As shown in Fig. 6, expression of p-JNK was increased in the farnesol-treated group compared to the control group. By contrast, p-ERK and p-p38 levels decreased as a result of farnesol treatment. These results indicate that farnesol induces apoptosis by regulating proteins related to the MAPK pathway.

To determine its effects on DU145 prostate tumor volume, farnesol was administered to groups of 3 mice for 5 weeks. After 5 weeks tumor volumes were $35 \%$ lower in the farnesoltreated group compared to the control group $(\mathrm{P}<0.05)$ (Fig. 7A). Farnesol also significantly reduced tumor weight (Fig. 7B). TUNEL-positive cell numbers were increased in tumors from mice treated with farnesol compared to the control mice (Fig. 7C). These results suggest that farnesol significantly reduced tumor size, weight, and increased TUNEL-positive cell numbers (Fig. 7). 
Collectively, the results of this study have shown farnesol to be a potential chemopreventive or therapeutic agent against DU145 prostate cancer.

\section{Acknowledgements}

This study was supported by the research grant of the Kongju National University in 2012.

\section{References}

1. Cohen LA: Nutrition and prostate cancer: a review. Ann NY Acad Sci 963: 148-155, 2002.

2. Kim EJ, Lim SS, Park SY, et al: Apoptosis of DU145 human prostate cancer cells induced by dehydrocostus lactone isolated from the root of Saussurea lappa. Food Chem Toxicol 46: 3651-3658, 2008.

3. Jain G, Voogdt C, Tobias A, et al: IкB kinases modulate the activity of the androgen receptor in prostate carcinoma cell lines. Neoplasia 14: 178-189, 2012.

4. Hellerstedt BA and Pienta KJ: The current state of hormona therapy for prostate cancer. CA Cancer J Clin 52: 154-179, 2002.

5. Siegel R, Naishadham D and Jemal A: Cancer statistics, 2012. CA Cancer J Clin 62: 10-29, 2012.

6. Rowan S, Rachet B, Alexe DM, et al: Survival from prostate cancer in England and Wales up to 2001. Br J Cancer 99 (Suppl 1) S55-S57, 2008.

7. Spitz MR, Strom SS, Yamamura Y, et al: Epidemiologic determinants of clinically relevant prostate cancer. Int J Cancer 89 259-264, 2000

8. Bostwick DG and Qian J: Effect of androgen deprivation therapy on prostatic intraepithelial neoplasia. Urology 58 (Suppl 1) S91-S93, 2001.

9. Ezekwudo D, Shashidharamurthy R, Devineni D, et al: Inhibition of expression of anti-apoptotic protein Bcl-2 and induction of cell death in radioresistant human prostate adenocarcinoma cell line (PC-3) by methyl jasmonate. Cancer Lett 270: 277-285, 2008.

10. Feldman BJ and Feldman D: The development of androgenindependent prostate cancer. Nat Rev Cancer 1: 34-45, 2001.

11. Jemal A, Siegel R, Ward E, et al: Cancer statistics, 2008. CA Cancer J Clin 58: 71-96, 2008.

12. Akao Y, Kusakabe S, Banno Y, et al: Ceramide accumulation is independent of camptothecin-induced apoptosis in prostate cancer LNCaP cells. Biochem Biophys Res Commun 294: 363-370, 2002

13. Akao Y, Banno Y, Nakagawa Y, et al: High expression of sphingosine kinase 1 and S1P receptors in chemotherapy-resistant prostate cancer PC 3 cells and their camptothecin-induced up-regulation. Biochem Biophys Res Commun 342: 1284-1290, 2006.

14. Rabi T and Bishayee A: $d$-Limonene sensitizes docetaxelinduced cytotoxicity in human prostate cancer cells: Generation of reactive oxygen species and induction of apoptosis. J Carcinog 8: 9, 2009.

15. Hanley K, Kömüves LG, Ng DC, et al: Farnesol stimulates differentiation in epidermal keratinocytes via PPARalpha. J Biol Chem 275: 11484-11491, 2000.

16. Rioja A, Pizzey AR, Marson CM and Thomas NS: Preferential induction of apoptosis of leukaemic cells by farnesol. FEBS Lett 467: 291-295, 2000

17. Bifulco M: Role of the isoprenoid pathway in ras transforming activity, cytoskeleton organization, cell proliferation and apoptosis. Life Sci 77: 1740-1749, 2005.

18. Miquel K, Pradines A and Favre G: Farnesol and geranylgeraniol induce actin cytoskeleton disorganization and apoptosis in A549 lung adenocarcinoma cells. Biochem Biophys Res Commun 225: 869-876, 1996.

19. Wright MM, Henneberry AL, Lagace TA, et al: Uncoupling farnesol-induced apoptosis from its inhibition of phosphatidylcholine synthesis. J Biol Chem 276: 25254-25261, 2001.

20. McTaggart SJ: Isoprenylated proteins. Cell Mol Life Sci 63: 255-267, 2006.

21. Ong TP, Heidor R, de Conti A, et al: Farnesol and geraniol chemopreventive activities during the initial phases of hepatocarcinogenesis involve similar actions on cell proliferation and DNA damage, but distinct actions on apoptosis, plasma cholesterol and HMGCoA reductase. Carcinogenesis 27: 1194-1203, 2006.
22. Goldstein JL and Brown MS: Regulation of the mevalonate pathway. Nature 343: 425-430, 1990.

23. Edwards PA and Ericsson J: Sterols and isoprenoids: signaling molecules derived from the cholesterol biosynthetic pathway. Annu Rev Biochem 68: 157-185, 1999.

24. Miquel K, Pradines A, Tercé F, Selmi S and Favre G: Competitive inhibition of choline phosphotransferase by geranylgeraniol and farnesol inhibits phosphatidylcholine synthesis and induces apoptosis in human lung adenocarcinoma A549 cells. J Biol Chem 273: 26179-26186, 1998.

25. Burke YD, Stark MJ, Roach SL, et al: Inhibition of pancreatic cancer growth by the dietary isoprenoids farnesol and geraniol. Lipids 32: 151-156, 1997.

26. Crowell PL: Prevention and therapy of cancer by dietary monoterpenes. J Nutr 129: S775-S778, 1999.

27. Chagas CE, Vieira A, Ong TP and Moreno FS: Farnesol inhibits cell proliferation and induces apoptosis after partial hepatectomy in rats. Acta Cir Bras 24: 377-382, 2009.

28. Bharate SB, Bhutani KK, Khan SI, et al: Biomimetic synthesis, antimicrobial, antileishmanial and antimalarial activities of euglobals and their analogues. Bioorg Med Chem 14: 1750-1760, 2006.

29. Katsuyama M, Ichikawa H, Ogawa S and Ikezawa Z: A novel method to control the balance of skin microflora. Part 1. Attack on biofilm of Staphylococcus aureus without antibiotics. J Dermatol Sci 38: 197-205, 2005.

30. McAnally JA, Jung M and Mo H: Farnesyl-O-acetylhydroquinone and geranyl-O-acetylhydroquinone suppress the proliferation of murine B16 melanoma cells, human prostate and colon adenocarcinoma cells, human lung carcinoma cells, and human leukemia cells. Cancer Lett 202: 181-192, 2003.

31. Burke YD, Ayoubi AS, Werner SR, et al: Effects of the isoprenoids perillyl alcohol and farnesol on apoptosis biomarkers in pancreatic cancer chemoprevention. Anticancer Res 22: 3127-3134, 2002

32. Tanida M, Niijima A, Shen J, et al: Olfactory stimulation with scent of lavender oil affects autonomic neurotransmission and blood pressure in rats. Neurosci Lett 398: 155-160, 2006.

33. Kikuzaki $\mathrm{H}$ and Nakatani N: Antioxidant effects of some ginger constituents. J. Food Sci 58: 1407-1410, 1993.

34. Duncan RE and Archer MC: Farnesol induces thyroid hormone receptor (THR) beta1 but inhibits THR-mediated signaling in MCF-7 human breast cancer cells. Biochem Biophys Res Commun 343: 239-243, 2006

35. Takahashi N, Kawada T, Goto T, et al: Dual action of isoprenols from herbal medicines on both PPARgamma and PPARalpha in 3T3-L1 adipocytes and HepG2 hepatocytes. FEBS Lett 514: 315-322, 2002.

36. Cantley LC: The phosphoinositide 3-kinase pathway. Science 296: 1655-1657, 2002.

37. Luo J, Manning BD and Cantley LC: Targeting the PI3K-Akt pathway in human cancer: rationale and promise. Cancer Cell 4: 257-262, 2003

38. Vivanco I and Sawyers CL: The phosphatidylinositol 3-Kinase AKT pathway in human cancer. Nat Rev Cancer 2: 489-501, 2002.

39. Schlieman MG, Fahy BN, Ramsamooj R, et al: Incidence, mechanism and prognostic value of activated Akt in pancreas cancer. Br J Cancer 89: 2110-2115, 2003.

40. Yamamoto S, Tomita Y, Hoshida Y, et al: Prognostic significance of activated Akt expression in pancreatic ductal adenocarcinoma. Clin Cancer Res 10: 2846-2850, 2004

41. Chadha KS, Khoury T, Yu J, et al: Activated Akt and Erk expression and survival after surgery in pancreatic carcinoma. Ann Surg Oncol 13: 933-939, 2006.

42. Terakawa N, Kanamori Y and Yoshida S: Loss of PTEN expression followed by Akt phosphorylation is a poor prognostic factor for patients with endometrial cancer. Endocr Relat Cancer 10: 203-208, 2003

43. Ng SS, Tsao MS, Nicklee T and Hedley DW: Wortmannin inhibits pkb/akt phosphorylation and promotes gemcitabine antitumor activity in orthotopic human pancreatic cancer xenografts in immunodeficient mice. Clin Cancer Res 7: 3269-3275, 2001.

44. Bondar VM, Sweeney-Gotsch B, Andreeff M, et al: Inhibition of the phosphatidylinositol 3'-kinase-AKT pathway induces apoptosis in pancreatic carcinoma cells in vitro and in vivo. Mol Cancer Ther 1: 989-997, 2002.

45. Fahy BN, Schlieman M, Virudachalam S and Bold RJ: Akt inhibition is associated with chemosensitisation in the pancreatic cancer cell line MIA-PaCa-2. Br J Cancer 89: 391-397, 2003. 
46. McCubrey JA, Lahair MM and Franklin RA: Reactive oxygen species-induced activation of the MAP kinase signaling pathways. Antioxid Redox Signal 8: 1775-1789, 2006.

47. Kholodenko BN and Birtwistle MR: Four-dimensional dynamics of MAPK information processing systems. Wiley Interdiscip Rev Syst Biol Med 1: 28-44, 2009.

48. Liu X, Li Q, Dowdell K, et al: Varicella-Zoster virus ORF12 protein triggers phosphorylation of ERK1/2 and inhibits apoptosis. J Virol 86: 3143-3151, 2012.

49. Junttila MR, Li SP and Westermarck J: Phosphatase-mediated crosstalk between MAPK signaling pathways in the regulation of cell survival. FASEB J 22: 954-965, 2008.

50. El-Deiry WS, Harper JW, O'Connor PM, et al: WAF1/CIP1 is induced in p53-mediated G1 arrest and apoptosis. Cancer Res 54: 1169-1174, 1994.

51. Dulić V, Kaufmann WK, Wilson SJ, et al: p53-dependent inhibition of cyclin-dependent kinase activities in human fibroblasts during radiation-induced G1 arrest. Cell 76: 1013-1023, 1994.

52. Yonish-Rouach E, Resnitzky D, Lotem J, et al: Wild-type p53 induces apoptosis of myeloid leukaemic cells that is inhibited by interleukin-6. Nature 352: 345-347, 1991.
53. Lowe SW, Schmitt EM, Smith SW, et al: p53 is required for radiation-induced apoptosis in mouse thymocytes. Nature 362 : 847-849, 1993.

54. Oda E, Ohki R, Murasawa H, et al: Noxa, a BH3-only member of the Bcl-2 family and candidate mediator of p53-induced apoptosis. Science 288: 1053-1058, 2000.

55. Nakano K and Vousden KH: PUMA, a novel proapoptotic gene, is induced by p53. Mol Cell 7: 683-694, 2001.

56. Sheu MJ, Chou PY, Huang CS, et al: Pipoxolan inhibits proliferation of HL-60 human leukaemia cancer cells by arresting the cell cycle at the G0/G1 phase. Clin Exp Pharmacol Physiol 37: 605-612, 2010.

57. Benson RS, Heer S, Dive C and Watson AJ: Characterization of cell volume loss in CEM-C7A cells during dexamethasoneinduced apoptosis. Am J Physiol 270: C1190-C1203, 1996.

58. Allen RT, Hunter WJ and Agrawal DK: Morphological and biochemical characterization and analysis of apoptosis. J Pharmacol Toxicol Meth 37: 215-228, 1997.

59. Prives C and Hall PA: The p53 pathway. J Pathol 187: 112-126, 1999. 